\title{
A Machine Learning-Based System for Detecting Leishmaniasis in Microscopic Images
}

\section{Mojtaba Zare}

Shiraz University of Medical Sciences

Hossein Akbarialiabad

Shiraz University of Medical Sciences

Hossein Parsaei

Shiraz University of Medical Sciences

Qasem Asgari

Shiraz University of Medical Sciences

Ali Alinejad

Shiraz University of Medical Sciences

Mohammad Saleh Bahreini

Shiraz University of Medical Sciences

Seyed Hossein Hosseini

Medical University of Ilam

Mohsen Ghofrani-Jahrom

Shiraz University of Medical Sciences

Reza Shahriarirad

Shiraz University of Medical Sciences

Yalda Amirmoezzi

Shiraz University of Medical Sciences

Sepehr Shahriarirad

Shiraz University of Medical Sciences

\section{Ali Zeighami}

Shiraz University of Medical Sciences

Gholamreza Abdollahifard ( $\nabla$ abdolahigh@sums.ac.ir)

Shiraz University of Medical Sciences

\section{Research Article}

Keywords: Leishmania, cutaneous Leishmaniasis, artificial intelligence, image processing, Adaboost, Viola-Jones, algorithm

Posted Date: August 31st, 2021 
DOl: https://doi.org/10.21203/rs.3.rs-677539/v1

License: (c) (1) This work is licensed under a Creative Commons Attribution 4.0 International License. Read Full License

Version of Record: A version of this preprint was published at BMC Infectious Diseases on January 12th, 2022. See the published version at https://doi.org/10.1186/s12879-022-07029-7. 


\section{A Machine Learning-based System for Detecting Leishmaniasis in Microscopic Images}

Mojtaba Zare ${ }^{1}$, Hossein Akbarialiabad ${ }^{2}$, Hossein Parsaei ${ }^{3,4}$, Qasem Asgari ${ }^{5}$, Ali Alinejad ${ }^{6}$, Mohammad

Saleh Bahreini ${ }^{7}$, Seyed Hossein Hosseini ${ }^{8}$, Mohsen Ghofrani-Jahrom ${ }^{9}$, Reza Shahriarirad ${ }^{10,11}$, Yalda Amirmoezzi $^{12,13}$, Sepehr Shahriarirad ${ }^{14}$, Ali Zeighami ${ }^{15}$, Gholamreza Abdollahifard ${ }^{16,17}$

1- Student Research Committee, Shiraz University of Medical Sciences, Shiraz, Iran (mojtabazarecb@gmail.com)

2- Student Research Committee, Shiraz University of Medical Sciences, Shiraz, Iran (Hosseinakbari7575@gmail.com)

3- Department of Medical Physics and Engineering, School of Medicine, Shiraz University of Medical Sciences, Shiraz, Iran (hparsaei@sums.ac.ir)

4- Shiraz Neuroscience Research Center, Shiraz University of Medical Sciences, Shiraz, Iran

5- Department of Parasitology and Mycology, School of Medicine, Shiraz University of Medical Sciences (asgarig@sums.ic.ir)

6- Student Research Committee, Shiraz University of Medical Sciences, Shiraz, Iran (alialinejad211@gmail.com)

7- Department of Medical Parasitology and Mycology, School of Medicine, Shiraz University of Medical Sciences, Shiraz, Iran (bahreinimohammadsaleh@gmail.com)

8- Department of Pediatrics, Ilam University of medical sciences, Ilam, Iran (shh6274412@gmail.com)

9- Department of Medical Physics and Engineering, School of Medicine, Shiraz University of Medical Sciences, Shiraz, Iran (mghofrani89@gmail.com) 
10- Thoracic and Vascular Surgery Research Center, Shiraz University of Medical Sciences, Shiraz, Iran (R.shahriari1995@gmail.com)

11- Student Research Committee, Shiraz University of Medical Sciences, Shiraz, Iran

12- Department of Medical Physics and Engineering, School of Medicine, Shiraz University of Medical Sciences, Shiraz, Iran (yaldaamirmoezzi@gmail.com)

13- Shiraz Neuroscience Research Center, Shiraz University of Medical Sciences, Shiraz, Iran

14- Student Research Committee, Shiraz University of Medical Sciences, Shiraz, Iran (shahriari77.s@gmail.com)

15- Student Research Committee, Shiraz University of Medical Sciences, Shiraz, Iran (ali.zeighami2009@gmail.com)

16- Department of community medicine, school of medicine. Shiraz university of medical sciences, Shiraz, Iran (abdolahigh@sums.ac.ir)

17- substance abuse and mental health research center, Shiraz University of medical sciences, Shiraz, Iran

\section{Corresponding Author:}

Dr. Gholamreza Abdollahifard M.D. (abdolahigh@sums.ac.ir)

Department of community medicine, school of medicine. Shiraz university of medical sciences, Shiraz, Iran

Substance abuse and mental health research center, Shiraz University of medical sciences, Shiraz, Iran

\section{Running title:}

\section{AI -based Leishmaniasis Detection System}




\begin{abstract}
Background: Leishmaniasis, a disease caused by a protozoan, causes numerous deaths in humans each year. After malaria, Leishmaniasis is known to be the deadliest parasitic disease globally. Currently, direct visual detection of Leishmania parasite through microscopy is the "gold standard" for the diagnosis of this disease. However, this method is time-consuming and subject to errors. This study was aimed to develop an artificial intelligence-based algorithm and image processing algorithms for the automatic diagnosis of Leishmaniasis.
\end{abstract}

Methods: The Viola-Jones algorithm was used in this study due to its high recognition speed. This algorithm performs in four stages: detection of Haar-like features, integral image creation, Adaboost training, cascade architecture.

Results: A $65 \%$ recall and $83 \%$ precision was concluded in the detection of macrophages infected with the Leishmania parasite. Also, these numbers were 52\% and $35 \%$, respectively, related to amastigotes outside of macrophages.

Conclusion: The results contain a fairly high sensitivity, with the specificity being less satisfactory. High processing speed, ease of work, and low expenses are advantages of the presented method compared to other procedures. By adding a few adjustments, this method could be considered a viable option.

Keywords: Leishmania, cutaneous Leishmaniasis, artificial intelligence, image processing, Adaboost, Viola-Jones, algorithm

\title{
Background
}


Leishmaniasis, a disease caused by more than 20 species of Leishmania parasites, is recognized in the tropical and subtropical regions as an acute disease with a high mortality rate. The disease manifests itself in both cutaneous and visceral forms and is transmitted via parasite-infected mosquitoes (1).

Cutaneous Leishmaniasis (CL) is endemic in more than 88 countries and around two-third of the cases occur in the countries Afghanistan, Algeria, Brazil, Pakistan, Peru, Saudi Arabia, Iran, and Syria(2, 3). Annually, CL has a prevalence of 0.7-1.2 million cases throughout the world(4). This zoonotic disease is caused by different strains in different countries in the world(5). In Iran, two species of $L$. tropica and L. major are the causative agents of CL, with approximately 20,000 cases reported each year(6). CL is estimated to be about 1.8\%-37.9\% in different rural provinces of Iran, with children being more affected(7). The clinical symptoms of CL vary depending on the species of the parasite, but usually, the disease begins with a papule or nodule, reaching its final size in about a week of slow growth. At the center of it, is a shell that may break apart and show a wound that will heal slowly over months or years(8). Papular and nodule-forming nodules account for $50 \%$ of cases, followed by plaque, impetiginized, and nodular forms. In a study by Dowlati et al., of the 4280 lesions observed, approximately $40 \%$ were on the face, $32 \%$ on the upper limb, $19.8 \%$ on the lower limb, and only $8.1 \%$ on the trunk $(9)$.

Due to certain diseases having similar clinical manifestations as Cutaneous Leishmaniasis (e.g. leprosy, skin cancer, tuberculosis, skin mycosis) and the wide clinical spectrum of CL, differential diagnosis of this disease rises in importance(10).

Nowadays, parasitological diagnosis is the gold standard method in the diagnosis of CL due to its high specificity. The process includes a microscopic examination of biopsies with Giemsa stained or aspirates, histological examination of fixed lesion biopsies, culture, triturates, or 
aspirates biopsy(10). Currently, microscopic examination is probably the most common diagnostic method because other techniques are expensive and are not available at the level of primary, secondary, and tertiary healthcare. Cultivation, as another method, provides a useful amount of information by which species can be identified and described with, but it is timeconsuming and requires expenditures and technical expertise. Besides, the sensitivity of this method is quite low (11).

Molecular parasitic diagnosis of CL has been extensively developed and reviewed over the past decade(12). Diagnosis is mainly performed by PCR-based methods and is particularly useful in cases of low parasitic multiplicity (e.g., mucosal Leishmaniasis). Additionally, treatment of CL patients can be controlled and followed up by this method. The specificity of this technique is 100, but the sensitivity of $20 \%$ to $30 \%$ in CL and $55 \%-70 \%$ in mucosal Leishmaniasis raise doubts when compared with conventional methods of parasite detection. Although much effort has been made to use molecular diagnostics in this area (e.g. successful discovery of parasitic DNA in blood or tissue stains; development of rapid PCR oligo-chromatography), its widespread use is still hampered due to the need for considerable laboratories' infrastructure, technical expertise, and costs (12).

In this paper, we developed an artificial intelligence (AI)-based decision support system to assist with resolving several issues associated with detecting and diagnosing Leishmania parasites under the microscope. Details of the method and its evaluation results using several real images are presented.

\section{Methods}


This study was conducted on 300 images taken from 50 laboratory slides acquired from lesions suspected of Leishmaniasis and from patients referred to Valfajr Clinic in Shiraz. These images include 150 photos from the 25 positive Leishmania slides and 150 photos from the 25 negative Leishmania slides (control). The slides were prepared and labeled by taking samples from inflamed edges of the wounds using a sterile scalpel and smeared on slides, followed by $100 \%$ ethanol fixation and Giemsa staining.

To design an intelligent system capable of detecting the infection in the collected smears according to the morphological data acquired by assessing the slides, the Viola-Jones algorithm is used. Briefly, the detector should be provided with images of both parasitic and non-parasitic samples so that it can gradually learn their distinctive features and become able to spot infected regions in an unseen image. Viola-Jones algorithm acts in the following steps: feature extraction, integral image creation, and classification. (13).

In a detection window, as shown in Figure 1, feature extraction consists of employing rectangles, whereas the sum of the pixels which lie within the white rectangles are subtracted from the sum of pixels in the grey rectangles. The difference in pixel intensities is then computed and used to categorize subsections of an image. Intuitively, these rectangle features are inspired by Haar wavelets which are simply square functions with various scales and translations. 


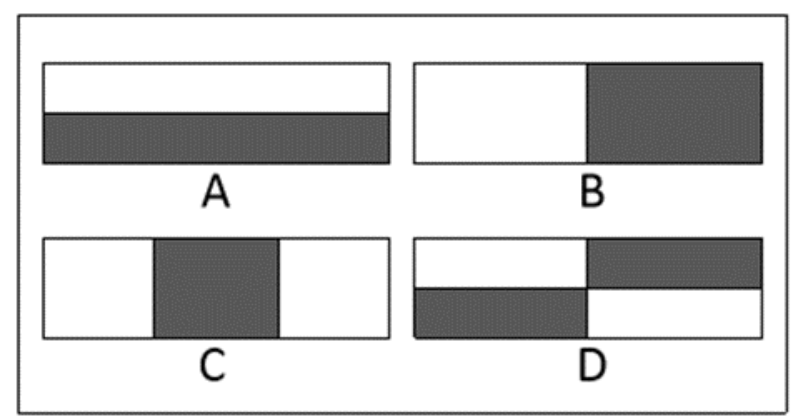

Figure 1. Rectangular window detection of Haar-like feature (14)

In any given image, irrelevant parts are highly likely to exist. Moreover, an abundant number of Haar-like features should be computed; to be precise, 162,336 features exist in a $24 \times 24$ pixel image window. To increase the processing speed significantly, it is proposed to compute an integral image, in which the intensity of each pixel at $(x, y)$ is the sum of all the pixels that reside above and to the left side.

By categorizing the subsections using Haar-like features, we can create integral images. The reason behind the categorization is to eliminate unwanted sections of our image and shorten processing time. To compute the sum of the pixel values in the subsections, array references are used. A single-rectangle sub-window needs four array references, while two, three, and four adjacent rectangle sub-windows need six, eight, and nine references, respectively. In an integral image of size $R \times C$, the main integral image $i i(R, C)$ is produced during single processing of the sum of the pixel values above and to the left of $(R, C)$. Once the integral image representation $i i$ of the original image $I$ is computed, the sum of original pixel values within any rectangle can be computed by a lookup table. Therefore, as shown in Figure 2, to compute the sum of pixel values in subsection $S_{1},\left(r_{1}, c_{1}\right)$ is needed and is computed as mentioned below: 
$\sigma\left(S_{1}\right)=i i\left(r_{1}, c_{1}\right)$

Whereas, to compute the values of subsection $S_{4}$, reference arrays $\left(r_{1}, c_{1}\right),\left(r_{2}, \mathrm{c}_{2}\right),\left(r_{3}, c_{3}\right)$, and $\left(r_{4}, \mathrm{c}_{4}\right)$ are needed.

$\sigma\left(S_{4}\right)=i i\left(r_{4}, c_{4}\right)-i i\left(r_{3}, c_{3}\right)-i i\left(r_{2}, c_{2}\right)+i i\left(r_{1}, c_{1}\right)$

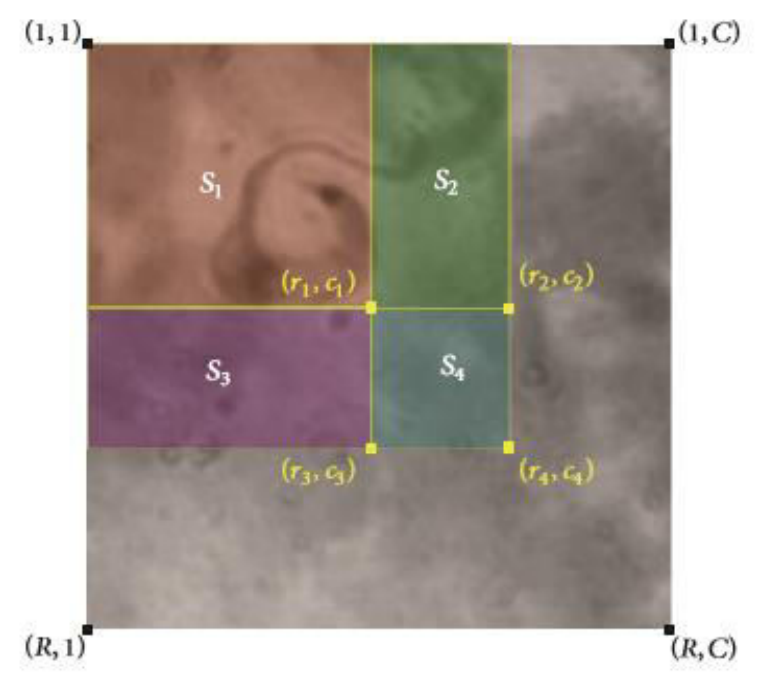

Figure 2. The complete expression of the integral image; the sum of the pixels inside subwindow $\mathrm{S}_{1}$ using the relation $\sigma\left(S_{1}\right)=i i\left(p_{1}\right)=i i\left(r_{1}, c_{1}\right)$ is obtained and also the sub-windows $\mathrm{S}_{2}$ and $\mathrm{S}_{3}$ with the relations $\sigma\left(S_{2}\right)=i i\left(r_{2}, \mathrm{c}_{2}\right)-\left(r_{1}, c_{1}\right)$ and $\sigma\left(S_{3}\right)=i i\left(r_{3}, c_{3}\right)-i i\left(r_{1}, c_{1}\right)$ are expressed. The pixels below $\mathrm{S}_{4}$ are also calculated as $\sigma\left(S_{4}\right)=i i\left(r_{4}, \mathrm{c}_{4}\right)-i i\left(r_{3}, c_{3}\right)-i i\left(r_{2}, c_{2}\right)+i i$ $\left(r_{1}, c_{1}\right)$. (Image by Uc-Cetina V. et al. (15))

Therefore, aside from creating integral images, certain learning algorithms are employed to select the best features and to train classifiers. Adaboost, currently the most popular boosting method, acts by adding weak learners to a boosted classifier one by one. This way, each new 
classifier is trained using a new set of information. The resulting classifiers are integrated with a cascade scheme.

Cascading is a stage-by-stage process, each stage consisting of a particular classifier with certain features. While all the features are grouped in these stages, the purpose of each stage is to determine whether a particular sub-window is not a match with the desired result or it may be a match; the desired result being the recognition of the previously defined morphological data. If a sub-window fails to find a match in any of the stages, it is discarded immediately. Therefore, usually, a classifier consisting of only a few simple and general features is used in the first stage/stages to rapidly remove unwanted subjects, granting more computational time to further stages requiring deeper analysis. Alternatively, a cascade of gradually more complex classifiers can achieve better detection rates, at the high cost of run-time speed, making it inefficient to do so. The sensitivity threshold can be adjusted in a cascade, preventing each stage from having a lower detection rate than the specified threshold. The total sensitivity will be the product of stage sensitivities. Ultimately, cascading classifiers enable the detection of the desired object in an image to be gradually approximated and a robust classifier is developed. Viola-Jones algorithm results in a drastic improvement of accuracy and execution time. The learning algorithm is as follows (15):

I. In a given dataset $\left(x_{1}, y_{1}\right) \cdot\left(x_{2}, y_{2}\right) \cdot \ldots \cdot\left(x_{n}, y_{n}\right), y_{i}$ is selected as 0 for negative cases and $y_{i}=1$ for positives.

II. For $y_{i}=0,1$ weights are considered to be $w_{1, j}=\frac{1}{2 m}, \frac{1}{2 l}$, respectively; where $m$ is the number of negative cases and $l$ the number of positives.

III. For $t=1, \ldots, T$ :

a) Weights are normalized as shown, 


$$
\frac{w_{t, i}}{\sum_{j=1}^{n} w_{t, j}} \rightarrow w_{t, i}
$$

b) Train the classifier $h_{j}$ for each $j^{\text {th }}$ feature, being restricted to using a single feature.

The error is computed using the equation $w_{t}, \epsilon_{j}=\sum_{i} w_{i}\left|h_{j}\left(x_{i}\right)-y_{i}\right|$.

c) The classifier with the lowest $\epsilon_{t}$ error, $h_{t}$, is chosen.

d) The weights are updated as:

$$
\begin{aligned}
& w_{t+1, i}=w_{t, i} \beta_{t}^{1-e_{i}}, \\
& \beta_{t}=\epsilon_{t} /\left(1-\epsilon_{t}\right) \text {, and } e_{i}=0,1 \text { for correct and incorrect classification of } \mathrm{x}_{\mathrm{i}} \text {, respectively. }
\end{aligned}
$$

IV. The strong classifier ensembled from single weak classifiers is as follows:

$$
\begin{gathered}
h(x)=\left\{\begin{array}{r}
1 \sum_{t=1}^{T} \alpha_{t} h_{t}(x) \geq \frac{1}{2} \sum_{t=1}^{T} \alpha_{t} \\
\text { otherwise }
\end{array}\right. \\
\text { Where } \alpha_{t}=\log \left(\frac{1}{\beta_{t}}\right)
\end{gathered}
$$

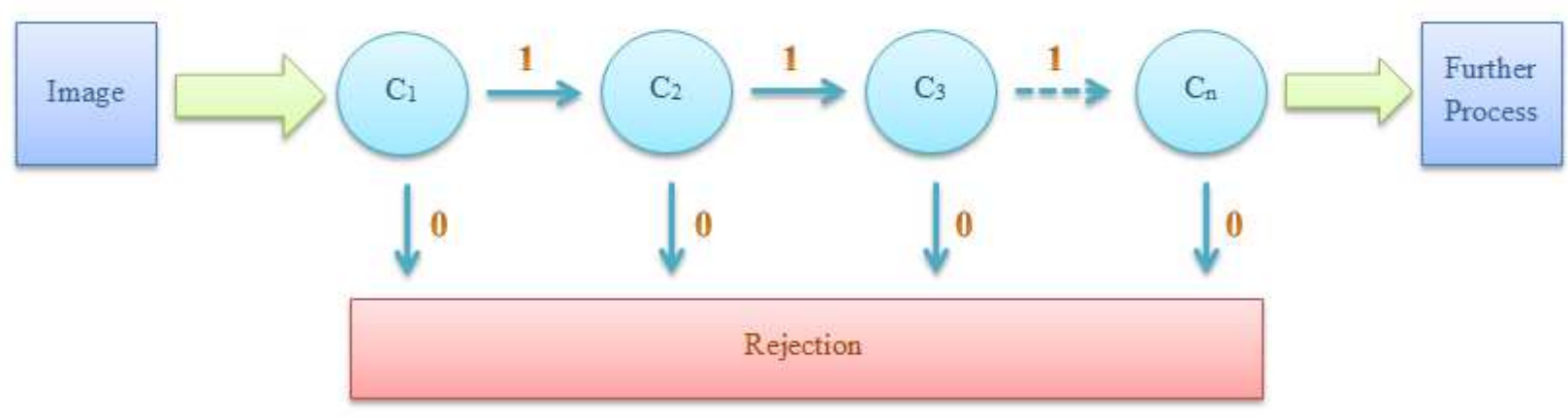

Figure 3. Adaptive boosting. The step-by-step process of tweaking classifiers 


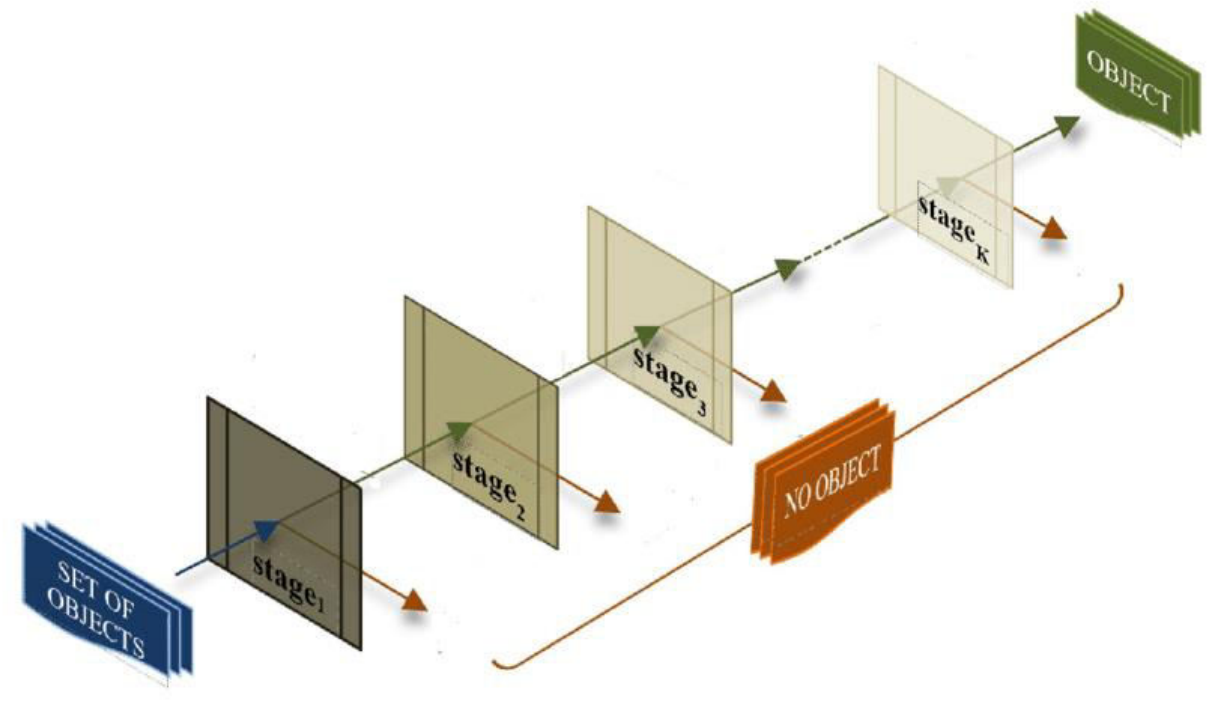

Figure 4. The performance of cascading classifiers (16)

\section{Results}

The performance of the developed system was quantitatively assessed using the two evaluation metrics Sensitivity and Specificity. Sensitivity is the probability of a positive test outcome in an infected patient, is calculated as shown below:

$$
\text { Sensitivity }=\frac{\text { True positive }}{\text { True positive }+ \text { False negative }}
$$


Therefore, the less the number of false negatives, the higher the sensitivity will be. In this study, this rate was computed to be $50 \%$ and $71 \%$ in infected macrophages and amastigotes outside of macrophages, respectively. (Figure 5)

Additionally, the chance of a negative test in a healthy patient, known as specificity, can be calculated similarly;

$$
\text { Specificity }=\frac{\text { True negative }}{\text { True negative }+ \text { False positive }}
$$

Meaning that a low count of false positives increases the likelihood of the method being specific and precise. Specificity in the detection of Leishmanial infected macrophages was shown to be $65 \%$, while it was $52 \%$ for individual parasites. (Figure 5)

Overall, when the output of the infected macrophages-based system and individual parasitesbased system were combined using OR combiner a sensitivity and specificity of $83 \%$ and $35 \%$ in parasite detection, respectively. (Figure 6) 


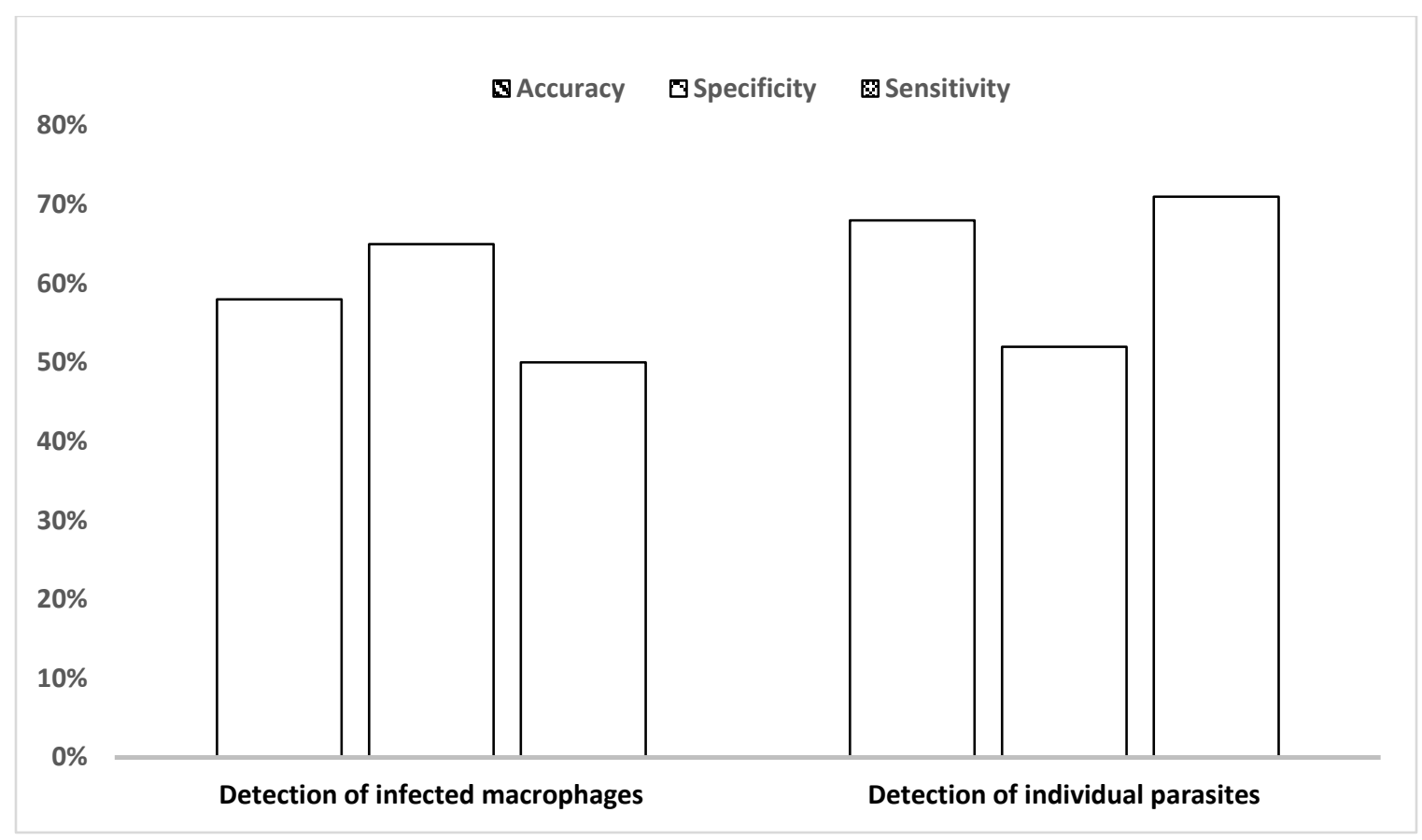

Figure 5. The accuracy, sensitivity, and specificity and of Leishmania detection system, both in and outside of macrophages.

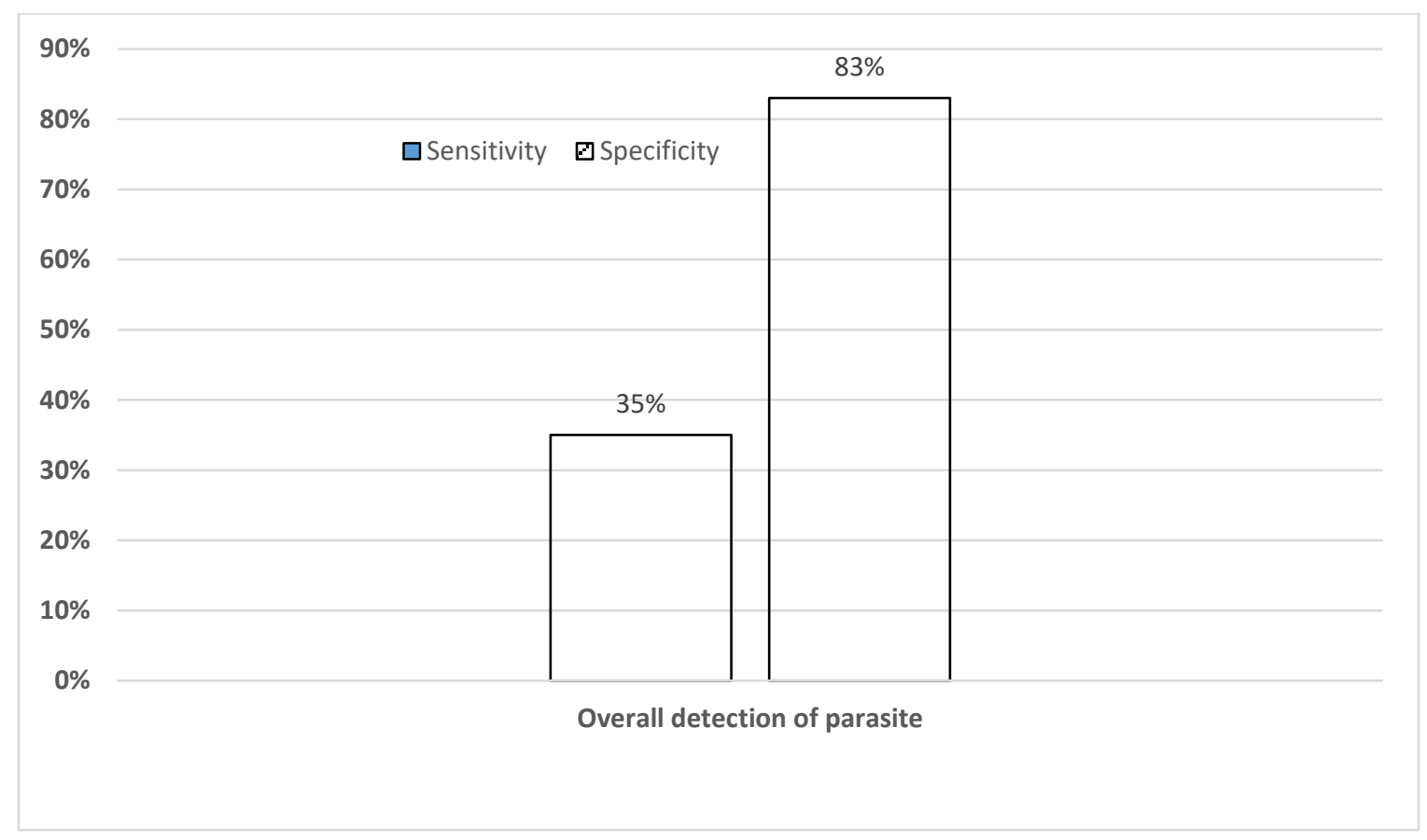

Figure 6. The sensitivity and specificity rates of Leishmania detection using mentioned methods 


\section{Discussion}

Automation can help prevent errors and mistakes caused by fatigue and tiredness due to long hours of working with microscopes and human error issues. By elimination of this factor, unnecessary prolongation of research will be prevented. At the moment, several countries, including Iran, possess microscopes that can be given certain algorithms and programs to act independently. These microscopes contain a high-resolution camera and an electromechanical plate, which is specially designed to navigate the blood smear according to the needs of the scan program. Another advantage of using this technology is the ability to control it from distance, granting the possibility of working from home.

In recent years, many methods have been used to diagnose the Leishmanial parasite. Although each method had been successful, they were replaced by improved and more efficient techniques. Direct visual recognition using a microscope is a simple and cost-efficient method for parasite detection; however, regardless of the skillfulness of the expert, it suffers from a relatively low sensitivity rate(17). Culture use requires its own set of tools and expenses, and the probability of infection with other microbial organisms during the process might negatively affect the aimed results of the test(18). Serological tests such as IFA and ELISA face the same issue as they cannot differentiate past and present infections. Additionally serological tests, due to low antibody titers of Leishmanial parasite, do not offer much value(19). Early diagnosis of deadly diseases, such as Leishmaniasis, results in an earlier treatment/control which can influence mortality rates significantly. Presently, PCR is known as the method presenting the highest sensitivity and specificity rates. In a study by Aviles et al., a result of $92 \%$ sensitivity and $100 \%$ specificity in cutaneous Leishmaniasis detection was achieved(19). Similar results were obtained in many other studies (20-23). However, in chronic cases, PCR sensitivity drops 
considerably(45.5\%)(20). Despite the promising results, PCR is a complex, expensive, and timeconsuming procedure requiring certain devices. Artificial intelligence and computer-aided diagnostic systems, to some extent, can be an answer to these problems. As shown in this study, with the help of the proposed framework sensitivity and specificities of 35\% and $83 \%$ were resulted in detecting CL using the Viola-Jones algorithm.

Many boosting methods have been developed over the years which can help learning methods and diagnostic systems work more efficiently. Adaboost, decision tree, KNN, linear regression, Naïve Bayes, Random Forest, and Extra tress are some of these methods. Saiprasath G et al. compared these 7 methods in an automated microscopic malaria detection procedure. The two methods, Random Forest and Adaboost proved to be more capable of generating desired results in terms of accuracy, sensitivity, specificity, and F1-score (24).

High recall rates are equivalent to a smaller count of false negatives. In deadly diseases such as Leishmaniasis, this percentage matters since infected patients should not be left unrecognized with the possibility of incorrectly being assumed healthy. Thus, necessary and deserved care and treatment can be provided, resulting in a lower morbidity and mortality rate. On the other hand, a high precision percentage indicates a low number of false positives. In some situations, the inadequacy of resources could prevent health experts from giving patients the care they need. A high number of false positives in a method could lead to unnecessary expenditure of resources and equipment and an increase in total expenses.

Bearing in mind the mentioned strengths and advantages of using intelligent diagnostic systems, keeping a heads-up in certain situations can help prevent any loss of efficiency. For example, if images acquired for the system contain low resolutions or have numerous dark parts (increased pixel count), the classification process would take more time, with the possibility of a greater 
number of false positives, thus overall efficacy drops. Moreover, these programs might need updates from time to time(25). F Thung et al., in efforts of finding an efficient method for automated detection of parasites, introduced Speeded-Up Robust Features (SURF). This procedure uses only images, without any need for learning and/or boosting algorithms. Unfortunately, the outcome proved to be unsatisfactory(25). Several procedures have shown to perform based on Image Segmentation(26). K-means clustering (27) and U-Net architecture are some techniques used in this process. Górriz $\mathrm{M}$ et al. achieved promising results using U-Net architecture for Leishmanial parasite detection(27). Despite the fantastic outcomes of this method, because of the need to process all features in an image, it is quite time-consuming (15 hours required by an NVIDIA GTX Titan x GPU)(27), while this procedure can be performed considerably faster using integral image creation and boosting methods such as Adaboost(15).

\section{Conclusion}

In this study, cutaneous Leishmaniasis detection was measured using a proposed diagnostic system. For this purpose, the Viola-Jones object detection algorithm enhanced by the Adaboost method was used. Results showed to have a fairly high specificity rate, whereas sensitivity rates did not. High processing speed, ease of use, and low expenses are advantages of this method. Lastly, by adding few adjustments such as providing the learning algorithm with a larger dataset, this method could be considered a viable option. 


\section{Abreviation:}

CL: Cutaneous Leishmaniasis

SURF: Speeded-Up Robust Features

\section{Declaration:}

- Ethics approval and consent to participate: not applicable

- Consent for publication: not applicable

- Availability of data and materials: not applicable

- Competing interests: The authors have no conflict of interest.

- Funding: no funding

Authors' contributions: All authors (MZ,HA,HP,QA,AA,MSB,SHH,MGJ,RS,YA,SS,AZ, and GA) had the same contribution. All authors have read and approved the manuscript.

- Acknowledgments: None. 


\section{References}

1. Organization WH. Control of the leishmaniases: report of a meeting of the WHO Expert Commitee on the Control of Leishmaniases, Geneva, 22-26 March 2010: World Health Organization; 2010.

2. Desjeux P. Leishmaniasis: current situation and new perspectives. Comparative immunology, microbiology and infectious diseases. 2004;27(5):305-18.

3. Norouzinezhad F, Ghaffari F, Norouzinejad A, Kaveh F, Gouya MM. Cutaneous leishmaniasis in Iran: results from an epidemiological study in urban and rural provinces. Asian Pacific journal of tropical biomedicine. 2016;6(7):614-9.

4. Alvar J, Vélez ID, Bern C, Herrero M, Desjeux P, Cano J, et al. Leishmaniasis worldwide and global estimates of its incidence. PloS one. 2012;7(5):e35671.

5. Guerrant RL, Walker DH, Weller PF. Tropical Infectious Diseases: Principles, Pathogens and Practice E-Book: Principles, Pathogens and Practice (Expert Consult-Online and Print): Elsevier Health Sciences; 2011. 
6. Mohammadiha A, Dalimi A, Mohebali M, Sharifi I, Mahmoudi M, Mirzaei A, et al. Molecular identification and phylogenetic classification of Leishmania spp. isolated from human cutaneous leishmaniasis in Iran: A cross-sectional study. Iranian Journal of Parasitology. 2018;13(3):351.

7. Rezaee N, Raissi V, Rajaeipour A, Nazari M, Getso M, Taghipour A, et al. Epidemiology, Associated Factors and Treatment Methods of Cutaneous Leishmaniasis Based on Previous Data from 2013 to 2018 in Ilam, Western Iran. Acta Parasitologica. 2020.

8. Hailu A, Dagne DA, Boelaert M. Leishmaniasis. Neglected Tropical Diseases-Sub-Saharan Africa: Springer; 2016. p. 87-112.

9. Dowlati Y. Cutaneous leishmaniasis: clinical aspect. Clinics in dermatology. 1996;14(5):425-31.

10. Escobar MA, Martinez F, Smith DS, Palma Gl. American cutaneous and mucocutaneous leishmaniasis (tegumentary): a diagnostic challenge. Tropical doctor. 1992;22(1_suppl):69-78.

11. Chargui N, Bastien P, Kallel K, Haouas N, Akrout FM, Masmoudi A, et al. Usefulness of PCR in the diagnosis of cutaneous leishmaniasis in Tunisia. Transactions of the Royal Society of Tropical Medicine and Hygiene. 2005;99(10):762-8.

12. Reithinger R, Dujardin J-C. Molecular diagnosis of leishmaniasis: current status and future applications. Journal of clinical microbiology. 2007;45(1):21-5.

13. Viola $\mathrm{P}$, Jones $\mathrm{M}$, editors. Rapid object detection using a boosted cascade of simple features. Proceedings of the 2001 IEEE computer society conference on computer vision and pattern recognition CVPR 2001; 2001: IEEE.

14. Viola P, Jones MJ. Robust real-time face detection. International journal of computer vision. 2004;57(2):137-54.

15. Uc-Cetina V, Brito-Loeza C, Ruiz-Piña H. Chagas parasite detection in blood images using AdaBoost. Computational and Mathematical Methods in Medicine. 2015;2015.

16. Golański P, Szczekala M. The analysis of the possibility of using Viola-Jones algorithm to recognise hand gestures in human-machine interaction. Prace Naukowe Instytutu Technicznego Wojsk Lotniczych. 2017;40(1):109.

17. Rodríguez N, Guzman B, Rodas A, Takiff H, Bloom BR, Convit J. Diagnosis of cutaneous leishmaniasis and species discrimination of parasites by PCR and hybridization. Journal of clinical microbiology. 1994;32(9):2246-52.

18. Lopez M, Inga R, Cueva N, Alvarez E, Arevalo J. PCR: a tool for diagnosis of American tegumentary leishmaniasis in a health post of rural endemic areas. Archives de l'Institut Pasteur de Tunis. 1993;70(3-4):499.

19. Aviles H, Belli A, Armijos R, Monroy FP, Harris E. PCR detection and identification of Leishmania parasites in clinical specimens in Ecuador: a comparison with classical diagnostic methods. The Journal of parasitology. 1999:181-7.

20. Vega-López F. Diagnosis of cutaneous leishmaniasis. Current opinion in infectious diseases. 2003;16(2):97-101.

21. Safaei A, Motazedian MH, Vasei M. Polymerase chain reaction for diagnosis of cutaneous leishmaniasis in histologically positive, suspicious and negative skin biopsies. Dermatology. 2002;205(1):18-24.

22. Ryan JR, Smithyman AM, Rajasekariah G-H, Hochberg L, Stiteler JM, Martin SK. Enzyme-linked immunosorbent assay based on soluble promastigote antigen detects immunoglobulin $\mathrm{M}$ (IgM) and IgG antibodies in sera from cases of visceral and cutaneous leishmaniasis. Journal of clinical microbiology. 2002;40(3):1037-43.

23. Hailu A. The use of direct agglutination test (DAT) in serological diagnosis of Ethiopian cutaneous leishmaniasis. Diagnostic microbiology and infectious disease. 2002;42(4):251-6.

24. Saiprasath G, Babu N, ArunPriyan J, Vinayakumar R, Sowmya V, Soman K. Performance comparison of machine learning algorithms for malaria detection using microscopic images. IJRAR; 2019. 
25. Thung F, Suwardi IS, editors. Blood parasite identification using feature based recognition.

Proceedings of the 2011 International Conference on Electrical Engineering and Informatics; 2011: IEEE. 26. Aimi Salihah A-N, Yusoff M, Zeehaida M. Colour image segmentation approach for detection of malaria parasites using various colour models and k-means clustering. 2013.

27. Górriz M, Aparicio A, Raventós B, Vilaplana V, Sayrol E, López-Codina D, editors. Leishmaniasis parasite segmentation and classification using deep learning. International Conference on Articulated Motion and Deformable Objects; 2018: Springer. 